\title{
Etudes pluridisciplinaires dans le domaine de l'environnement - Le système TELEMAC -
}

\author{
Environmental multi-disciplinary studies - The TELEMAC system
}

\author{
par J.-M. Hervouet \\ Groupe Hydraulique Fluviale \\ Laboratoire National d'Hydraulique et Environnement. \\ Direction Recherche et Développement. \\ Electricité de France.
}

With the example of the Telemac system, different aspects of the development of hydro-informatic systems by multidisciplinary teams are reviewed in this article. Some technical solutions such as structured programming, external coupling, and automatic generation of source code are briefly sketched. An example of bridge between two very different disciplines, astrophysics and hydraulics, the so-called SPH method is given.

\section{I $\square$ INTRODUCTION}

Le mouvement brownien, dont l'étude devait lancer la carrière d'un employé de l'office fédéral des brevets de Berne nommé Albert Einstein, fut découvert par le botaniste écossais Robert Brown, dont l'œuvre principale est une flore d'Australie : "Prodromus Florae Novae-Hollandiae".

La théorie du chaos [1], une des percées scientifiques majeures de la fin du vingtième siècle, fut portée sur les fonds baptismaux par, entre autres, un météorologue (Edward Lorenz), un chercheur d'IBM intéressé par la fluctuation des marchés (Benoît Mandelbrot), un mathématicien du laboratoire de Los Alamos passionné par les phénomènes non-linéaires (Mitchell Feigenbaum), et un spécialiste du comportement quantique de l'hélium superfluide à l'Ecole Normale Supérieure (Albert Libchaber).

On pourrait multiplier ainsi à l'infini les exemples, la pluri-disciplinarité est d'évidence féconde, et beaucoup de découvertes et d'innovations se font aujourd'hui aux interfaces entre spécialités, au sein de nouvelles sciences syncrétiques aux noms futuristes comme la bionique, l'ethnobotanique ou autres néologismes. Les progrès ne découlent souvent plus d'une nouvelle loi de la physique ni même parfois d'une avancée technologique mais simplement d'une organisation différente, d'une utilisation différente de techniques déjà connues, d'un autre regard. Les télescopes et les accélérateurs de particules ne sont plus les voies royales et uniques d'amélioration de la connaissance. Le temps s'éloigne où l'on pouvait dire "Parmi les sciences, il y a la physique et puis il y a la chimie qui est une sorte de physique, et puis il y a les collections de timbres". Cette classification péremptoire de Lord Rutherford sonne aujourd'hui de plus en plus comme le malheureux "Racine passera, comme le café" de la marquise de Sévigné. Toute la science ne se réduit pas à la mécanique quantique, connaitre la physique des particules n'est pas comprendre ses infinies déclinaisons dans la nature, énoncer les équations de NavierStokes n'est pas expliquer la turbulence.

Certes, l'époque héröque des Léonard de Vinci et des Pic de la Mirandole est révolue et le progrès scientifique est bien l'affaire de spécialistes, mais de spécialistes groupés en équipes, et d'équipes qui communiquent. L'étude des problèmes d'environnement est par excellence un de ces champs de pluridisciplinarité où se mêlent entre autres l'hydraulique, la chimie, la biologie, qui elles-mêmes font appel aux mathématiques, à la métrologie, à l'informatique et à la modélisation numérique, sans parler d'économie, de politique et de sociologie. En environnement, la pluridisciplinarité n'est pas seulement une fructueuse méthode heuristique pour susciter l'innovation, un changement de paradigme créateur d'idées, elle est simplement l'incontournable adaptation à la réelle complexité du monde. A Electricité de France, après une période pionnière de grands aménagements où primait l'hydraulique pure, nous sommes aujourd'hui de plain-pied avec les questions de développement durable et d' "acceptabilité", dans une approche intégrée et globale.

L'objectif du présent article est de montrer, après l'exposé de quelques exemples, comment les différentes disciplines intervenant dans l'hydraulique de l'environnement peuvent communiquer et s'articuler au sein d'un système hydroinformatique, comment la maintenance et la maîtrise de tels systèmes devient en elle-même un nouveau défi. Nous pren- 
drons l'exemple du système Telemac développé à Electricité de France. Après avoir fait une brève description de ses composantes et de ses applications (une présentation plus détaillée figure dans [2]) nous mettrons l'accent sur les particularités de génie logiciel, de structuration et de fonctionnement développées pour favoriser la communication, le travail et les échanges au sein d'équipes pluridisciplinaires. Nous verrons successivement la structuration de la programmation, le couplage de codes, la génération automatique de modèles de qualité de l'eau, l'organisation des co-développements. Enfin nous mentionnerons une nouvelle technique numérique, la méthode SPH (Smoothed Particle Hydrodynamics), bel exemple de multidiscipinarité puisqu'elle émane de l'astrophysique.

\section{II ם BRÈVE PRÉSENTATION DU SYSTÈME TELEMAC}

\subsection{Les composantes du système}

Le système Telemac est un ensemble de logiciels de simulation numérique dédiés aux problèmes d'hydraulique de l'environnement. D'abord appliqué aux écoulements à surface libre, il couvre maintenant également les écoulements souterrains. Initialement développé entièrement par Electricité de France, il bénéficie aujourd'hui d'une diffusion et d'accords de co-développement qui en font non seulement un outil standard d'ingéniérie mais aussi une plate-forme de recherche de portée internationale.

Le système est composé d'une part de pré et post-processeurs (mailleurs et logiciels de dessin qui permettent la saisie des données et l'exploitation des résultats), d'autre part d'une série de logiciels de simulation fondés principalement sur la technique des éléments finis et adaptés chacun à un champ d'applications particulier.

Les principaux atouts du système sont la flexibilité apportée par la méthode des éléments finis qui s'appuie sur des maillages non structurés, la puissance des algorithmes développés au sein d'une bibliothèque commune d'outils dans laquelle puise chaque programme, et de façon générale les différents facteurs d'échelle apportés par la mise en commun des pré- et post-processeurs et l'architecture commune des logiciels, aussi bien dans les phases de maintenance et de développement que dans les phases d'apprentissage.

Le mailleur Matisse est fondé sur la technique de triangulation de Delaunay sous contrainte. Les maillages sont générés automatiquement à partir d'un modèle numérique de terrain et en fonction de critères de taille de maille donnés par l'utilisateur, et de contraintes qu'il impose : points et lignes que l'on veut voir figurer dans le maillage. La géométrie algorithmique utilisée dans Matisse a été mise au point à l'Ecole des Mines de Saint-Etienne et est fondée sur une notion innovante appelée "arithmétique paresseuse" qui permet de maîtriser de façon optimale les classiques problèmes de troncature des ordinateurs.

Le logiciel de visualisation Rubens permet de composer à volonté des figures avec des champs de vitesses, des surfaces colorées, des vues en perspective, des textes, des profils. Il peut fournir entre autres des fichiers au format PostScript.

Parmi les programmes de simulation numérique proprement dits, nous trouvons d'abord les codes d'hydrodynamique Telemac-2D et Telemac-3D (voir aussi [3]). Le pre- mier résout les équations de Saint-Venant ; il fournit en tout point d'un maillage la hauteur d'eau et un vecteur vitesse moyenné sur la verticale. Ces applications sont principalement le calcul des crues et des inondations, les marées et la simulation des ondes de rupture de barrage. Telemac-3D résout en dimension 3 les équations de Navier-Stokes complètes, avec ou sans hypothèse hydrostatique. Il s'applique à des écoulements plus complexes où les phénomènes de densité ou d'accélération verticale prennent plus d'importance.

La connaissance des courants et des hauteurs d'eau permet ensuite d'utiliser les logiciels de sédimentologie et de qualité de l'eau. Sysiphe, co-propriété du CETMEF (ministère des Transports), d'EDF et de SOGREAH, simule le transport de sédiments par charriage sur le fond. Subief-2D et Subief-3D reproduisent en dimension 2 et 3 le transport de sédiments en suspension et calculent le devenir d'un nombre quelconque de polluants qui peuvent interagir.

Un ensemble de modèles (Artémis, Tomawac et Cowadis), sont dédiés à l'étude des vagues et à leur génération par le vent. Enfin Estel-2D et Estel-3D reproduisent les écoulements souterrains, ils sont l'équivalent pour ce milieu de Telemac-2D et 3D. Une bonne surprise de la pluridisciplinarité est d'ailleurs qu'une analogie entre hauteur d'eau et porosité permet d'utiliser nos logiciels de qualité de l'eau aussi bien en surface libre qu'en écoulement souterrain.

Signalons enfin que le système Telemac est diffusé par des grands laboratoires d'hydraulique : Sogreah en France, HRWallingford en Angleterre et le Centre Hydraulique Canadien du National Research Council au Canada.

\subsection{Les applications}

Il y a maintenant des centaines d'applications de Telemac de par le monde : nous nous restreindrons à citer quelquesunes de celles où intervient actuellement le Laboratoire National d'Hydraulique et Environnement (LNHE) et qui illustrent le mieux la pluridisciplinarité.

Le projet Rescdam : il s'agit d'un projet européen avec l'ENEL et des partenaires finlandais dont le "Finnish Environment Institute" et l'Université d'Helsinki. Le sujet est la constitution d'un plan d'évacuation en cas de rupture de barrage et le conseil aux populations. Le site pilote du barrage de Seïnajoki en Finlande a été choisi. A côté de sujets classiques comme la simulation numérique d'une onde de rupture, des expériences en canal de résistance au courant de sujets d'âges et de poids divers ont été réalisés pour préciser la notion de condition d'écoulement dangereuse. La présentation des résultats de calcul, cartes et animations, aux populations et aux autorités de sûreté prend dans un tel projet une grande importance. Toutes les techniques nouvelles des Systèmes d'Information Géographiques sont en conséquence pleinement exploitées.

L'envasement de l'estuaire de la Rance : cette étude a pour but de reproduire les mécanismes hydro-sédimentaires à l'œuvre dans l'estuaire de la Rance, et d'examiner des scénarios pour limiter son envasement et sa pollution. Le client est le Conseil Opérationnel des Elus et Usagers de la Rance (COEUR). Sogreah et le groupe de consultants Hydroexpert sont partenaires du LNHE. L'étude couple la courantologie, la sédimentologie, la qualité de l'eau (des rejets de coliformes fécaux sont simulés), mais les intérêts économiques sont bien entendu aussi à prendre en compte. Diverses solutions sont envisagées qui vont de l'installation de pièges à sédiments à un fonctionnement optimisé de l'usine marémo- 
trice.

L'aval du cycle : l'étude quantitative de la migration de polluants dans les milieux géologiques est au cœur de nombreux enjeux environnementaux importants pour EDF : stockage profond de déchets nucléaires, contaminations de sols par des métaux lourds ou des polluants organiques issus d'activités industrielles. Les études d'impact associées à ces problèmes nécessitent souvent l'utilisation d'outils numériques pluri-disciplinaires capables de prendre en compte des phénomènes à la fois hydrodynamiques et géochimiques. Dans le cadre de partenariats avec des laboratoires universitaires, de telles fonctionnalités ont été introduites dans le système Telemac :

- le module Estel-2D ou 3D (développé en partenariat avec l'Université de Bristol) permet de simuler des écoulements souterrains en dimensions 2 ou 3 dans un milieu saturé ou non saturé.

- le module Subief utilise les écoulements générés par Estel afin de transporter les polluants étudiés et peut être couplé (voir le paragraphe IV) avec le module géochimique CHESS, développé par l'Ecole des Mines de Paris, qui permet de prendre en compte la réactivité chimique des polluants avec les autres espèces aqueuses ou bien avec les phases minérales constituant le milieu géologique.

La qualité de l'eau dans la Seine à Achères : Telemac est utilisé dans le cadre du groupe pluridisciplinaire PIRENSeine, pour modéliser les phénomènes biologiques dans la vase. L'étude fait l'objet d'une thèse au Centre d'Informatique Géologique de l'Ecole des Mines de Paris. La mise au point d'un modèle dit "benthique" requiert une dizaine de variables physiques et biologiques telles que les bactéries, l'oxygène dissous, la matière organique particulaire et le phytoplancton. Des campagnes de mesures accompagnent et valident la recherche. Le traceur choisi pour la validation est original ; il s'agit de la propriété de conductivité électrique de l'eau, reflet de l'ensemble des traceurs transportés.

\section{STRUCTURATION DE LA PROGRAMMATION}

Il est utopique d'espérer que dans une équipe pluridisciplinaire chacun devienne spécialiste de tout, ce n'est pas le but. L'enjeu est plutôt d'autoriser l'ignorance, de cacher la complexité à ceux qui n'en ont pas besoin. Il y a là un sujet délicat et les puristes clameront haut et fort que l'utilisation de boîtes noires ne peut mener qu'à des catastrophes. Certains programmeurs sont même fiers d'une certaine opacité qui décourage, disent-ils, les Béotiens. Certes, il y a danger d'usage à mauvais escient mais peut-on en rester à ce constat élitiste aux relents de corporatisme ? Mieux vaut travailler l'ergonomie et la programmation défensive, structurer en vue d'une utilisation à différents niveaux d'expertise. L'ensemble du système Telemac repose sur une bibliothèque d'éléments finis appelée Bief, dans laquelle puise chaque programme de simulation. Des versions successives de la bibliothèque apportant diverses améliorations sont proposées périodiquement et les responsables de chaque code sont alors chargés de s'adapter aux modifications. L'intérêt de cette structuration est que chaque développeur de code peut se consacrer à la physique de ces équations sans connaître tous les arcanes de la méthode des éléments finis et du génie logiciel.

Une phase-clef de l'évolution du système a été le passage à un langage structuré, en l'occurrence le Fortran 90. La création de structures permet l'organisation en couches de logiciel qui représentent chacune un niveau de complexité. On peut distinguer 4 niveaux successifs : l'utilisateur novice préparant un cas simple pilotera les programmes à partir d'un tableau de bord de paramètres physiques et numériques comme la viscosité moléculaire ou le nombre de pas de temps. Nul besoin de programmation à ce niveau. L'utilisateur confirmé aura de plus accès à un jeu de sous-programmes qu'il peut modifier pour les adapter à son cas particulier. Cette ouverture du système, même si elle requiert des connaissances en programmation, est hautement appréciée, voire exigée, par les utilisateurs. Le troisième niveau est celui du développeur de code. A l'aide d'un guide de programmation de la bibliothèque Bief, il peut modifier ses équations, enrichir et développer son programme en utilisant les structures de matrice, de vecteur et les divers outils lui permettant de naviguer dans un maillage d'éléments finis. Résoudre un système linéaire n'est pour lui qu'un appel au sous-programme SOLVE. Seules sont requises à ce niveau les connaissances théoriques qui mènent depuis la forme continue des équations jusqu'à leur expression discrète sous forme de systèmes linéaires. Le quatrième niveau est celui du développeur de la bibliothèque Bief ; c'est le seul où apparaissent les détails des méthodes de résolution de systèmes linéaires et du préconditionnement, les techniques de stockage de matrices creuses, etc. L'utilisation d'un langage structuré où l'on peut définir de nouveaux objets ou changer leurs caractéristiques rend transparentes la plupart des modifications effectuées à ce quatrième niveau. Par exemple, la découverte en 1999 d'un nouveau mode de stockage des matrices, le stockage par segment, a permis de réduire le coût calcul de certains des logiciels du système de $40 \%$, sans en changer une seule ligne. Les changements induits n'apparaissaient en effet que dans des composantes de structures qui n'étaient utilisées que dans la bibliothèque Bief.

Le Fortran 90 est un langage structuré mais, objecteront certains, ce n'est pas un langage de programmation par objets ; pourquoi l'avoir choisi plutôt que le C++ qui est beaucoup plus répandu? Tout simplement pour des raisons historiques parce que les logiciels scientifiques ont été à l'origine écrits en Fortran 77 . Un passage à $\mathrm{C}++$ ou même Java est envisageable mais dans le cas de Telemac il fallait trouver un chemin critique permettant de sortir chaque année une nouvelle version validée. Dans un premier temps, les structures ont été simulées avec des fonctions en Fortran 77 (version 4.0 du système), dans un deuxième temps la véritable migration vers le Fortran 90 a mené à la version 5.0. Cette dernière migration a pris selon les codes de une à deux semaines de travail. Cet investissement est rentable car l'on tend maintenant vers une compatibilité entre versions successives de Bief qui assure une évolution des codes de simulation sans aucun travail d'adaptation. Tous les programmeurs constatent une plus grande lisibilité et concision des nouvelles versions. Le code source de Bief fait maintenant environ 75000 lignes, commentaires compris, le code source de Sysiphe fait 6500 lignes. L'ensemble du système est donc resté à échelle humaine et l'on est loin de la taille gigantesque des programmes de télécommunication ou des systèmes d'exploitation dont la maintenance devient rapidement problématique. 


\section{IV — COUPLAGE EXTERNE DES CODES}

L'organisation en système hydro-informatique, avec des modules de petite taille, a des avantages évidents pour le développement et la maintenance, et permet des interventions ponctuelles qui ne déclenchent pas de grands tremblements de terre dans l'ensemble. Il faut maintenant organiser la communication entre les éléments. La direction Recherche et Développement d'EDF a choisi pour cela le couplage dit "externe" avec la construction du logiciel CALCIUM qui permet à différents programmes de communiquer entre eux. CALCIUM est fondé sur les techniques de parallélisme, plus précisément sur le langage PVM. Les programmes communiquent entre eux par des ordres spéciaux de lecture et d'écriture. La nature et la taille des échanges (entiers, réels,...) est spécifiée dans un tableau de bord. CALCIUM se charge du lancement de chaque programme et organise les points de rendez-vous. L'économie en temps de développement est considérable. Sans cette notion de couplage externe, le système Telemac ne serait qu'un unique programme tentaculaire et ingérable car le couplage est partout, il est l'expression même de la pluridisciplinarité. Il faut coupler hydrodynamique et sédimentologie car les évolutions du fond changent les courants et inversement. Il faut coupler la houle et les courants, il faut coupler le charriage de sédiments sur le fond et le transport en suspension. Il faut coupler écoulements souterrains et cinétique chimique rapide. Le couplage pas de temps par pas de temps de Telemac-2D et Subief-2D dans le cas des prises d'eau des centrales nucléaires en bord de mer a en particulier montré un effet spectaculaire : si l'on calcule les courants pendant une marée, en supposant le fond fixe, et qu'on déduit a posteriori l'évolution sédimentaire pendant la même période, on "prédit", pour un ensemble de conditions qui correspondent à un cas réel, un trou de $15 \mathrm{~m}$ dans le sable ! Si l'on répercute à chaque pas de temps les évolutions du fond dans l'hydrodynamique, le trou ne fait plus que $15 \mathrm{~cm}$. Les relations interdisciplinaires s'en trouvent notablement améliorées : plus de soupçons de "mauvais" courants ou de "mauvaises équations de transport”, les déboires d'antan n'étaient qu'un problème de communication.

Le couplage externe et CALCIUM ont trouvé une autre application avec l'identification de paramètres. Il s'agit de trouver des données manquantes, par exemple des coefficients de frottement sur le fond, en fonction d'événements connus, comme des crues. L'opération suppose un certain nombre d'essais et erreurs avec un code (par exemple Telemac-2D) et son code adjoint, une sorte de jumeau requis par la théorie du problème inverse. CALCIUM organise les appels du code et de son adjoint, qui peuvent ainsi être maintenus et évoluer séparément.

\section{GÉNÉRATION AUTOMATIQUE DE CODE SOURCE}

Même si tout est fait pour faciliter, ou éviter, la programmation avec l'organisation de la bibliothèque Bief, adapter le système à de nouvelles équations exige bien entendu la création d'un nouveau module et des connaissances en analyse numérique. Peut-on envisager de soulager des spécialistes d'horizons divers de cette étape rébarbative, voire rédhibitoire et offrir une programmation automatique de la résolution des équations ? Ce sujet futuriste a reçu dans le système
Telemac un début de solution, au moins pour les modèles de qualité de l'eau. Dans ce cas la forme des équations est toujours la même et représente le transport d'une concentration, d'un traceur ou d'un polluant, et sa diffusion, avec des termes de création et de destruction. Les traceurs peuvent interagir mais ce couplage peut être fait de façon explicite. Avec ces hypothèses il a été possible d'écrire un logiciel nommé WQ2SUBIEF capable d'interprêter un fichier ASCII où l'on décrit les équations d'un modèle de qualité d'eau et de le retranscrire en lignes de Fortran qu'il ne reste plus qu'à insérer dans des sous-programmes de Subief-2D prévus à cet effet. Il y a là un découplage complet entre la définition et la mise au point d'un modèle de qualité de l'eau, le choix des variables et des constantes physiques d'une part, sujet sensible où, disent les spécialistes, tous les cas sont particuliers, et, d'autre part, la résolution numérique. La principale difficulté reste l'interprétation des résultats et la recherche d'erreurs. L'expérience montre dans ce domaine que les physiciens ont une tendance naturelle à chercher des explications physiques à un artefact numérique. L'inverse est plus rare mais, pour ne citer qu'un exemple, l'oubli du phénomène de pente limite d'un tas de sable en sédimentologie produit des résultats qui ressemblent fort à une erreur numérique.

\section{VI $\square$ PLURIDISCIPLINARITÉ ET COOPÉRATION INTERNATIONALE}

Petit à petit et grâce à sa diffusion à l'extérieur d'EDF, d'abord au sein de grands organismes et d'universités, et aujourd'hui aussi dans de petits bureaux d'études, le système Telemac a vu se constituer autour de lui une équipe pluridisciplinaire et internationale. Des avancées techniques importantes, comme une version non-hydrostatique de Telemac$3 \mathrm{D}$, des développements en parallélisme, des idées sur le traitement des zones sèches et des stratifications, viennent maintenant enrichir l'ensemble, en provenance des services de navigation allemands, des universités de Hanovre et de Bristol, du Service Hydrographique et Océanographique de la Marine, et de bien d'autres. Plus de soixante équipes de scientifiques et d'ingénieurs contribuent, par leur utilisation, par leurs remarques, à la validation et au progrès de l'ensemble. Etre au carrefour de ces développements est une tâche fascinante où l'on voit toute la puissance du travail d'équipe, mais où l'on en saisit aussi toute la difficulté. Fournir une documentation à jour et en anglais, répercuter les corrections d'erreurs, conseiller, "encapsuler" la connaissance dans les logiciels, modifier sans déstabiliser, réunir les utilisateurs, autant de tâches qui ne se justifient qu'avec une diffusion d'ampleur suffisante. Le génie logiciel se teinte au passage de négociation internationale et de gestion de projet avec des équipes dispersées. Le Software devient Peopleware $[4,5]$.

\section{VII — DE L'ASTROPHYSIQUE À L'HYDRAULIQUE}

La pluridisciplinarité peut se concevoir à différentes échelles. Plus on progresse dans une spécialité, plus on remarque de différences que l'on ne soupçonnait pas, plus le discours devient jargon. Mettez en présence un spécialiste des éléments finis et un tenant de la méthode des volumes finis et vous comprendrez que la pluridisciplinarité commence avec votre voisin de bureau. La spécialisation est 
donc paradoxalement aussi créatrice de pluridisciplinarité et donne de la communauté scientifique une image fractale que ne renierait pas le déjà nommé Benoît Mandelbrodt. Avec la méthode SPH (Smoothed Particle Hydrodynamics), point de pluridisciplinarité de voisinage, ou à petit grain. $\mathrm{La}$ méthode vient d'Australie, et de I'astrophysique. Après avoir fait des modèles d'accrétion de la lune et de formation des étoiles, le professeur Monaghan s'est soudain avisé que sa nouvelle méthode particulaire pouvait aussi bien résoudre les équations de NavierStokes, avec une souplesse qui apporte des espoirs nouveaux à de vieux problèmes : les écoulements diphasiques, les écoulements mixtes qui couplent surface libre et passage en charge, la stabilité des digues et bien d'autres. Dans le cadre du projet Telemac, une maquette de logiciel a été construite pour essayer cette méthode. En quelques mois le résultat de la figure 1 , l'impact d'une vague sur un mur vertical, a pu être obtenu. Le franchissement d'un seuil, le mélange de deux couches de densités différentes, un courant de turbidité, un déferlement de vague ont ensuite montré tour à tour l'énorme potentiel de la technique et un prototype industriel est maintenant en construction. En l'occurrence, c'est l'astrophysique qui est venue à l'hydraulique. Peut-être aurait-il fallu, pour gagner quelques années, être abonné à l'Astronomical Journal. Sans aller jusqu'à recommander de lire la flore d'Australie de Robert Brown, voici qui élargit quand même sensiblement le champ d'action de la veille technologique.

\section{VIII $\square$ CONCLUSION}

Les systèmes hydro-informatiques utilisés dans les problèmes d'hydraulique de l'environnement rassemblent et cristallisent aujourd'hui un ensemble de connaissances émanant d'un grand nombre de disciplines. Ils sont devenus à la fois des outils de calcul, des systèmes experts et des encyclopédies numériques. Leur possession et leur maîtrise deviennent des enjeux stratégiques. Leur maintenance et leur développement posent des problèmes nouveaux d'organisation et de collaboration internationnale et l'on assiste au développement d'une ingéniérie de la pluridisciplinarité. A l'heure du village planétaire et des fusions d'entreprises, compétition et concentration sont à prévoir et il faudra être dans les premiers pour durer. Le progrès n'est plus dans l'établissement des équations, il porte maintenant sur la

\section{Methode SPH : 'Smoothed Particle Hydrodynamics'}
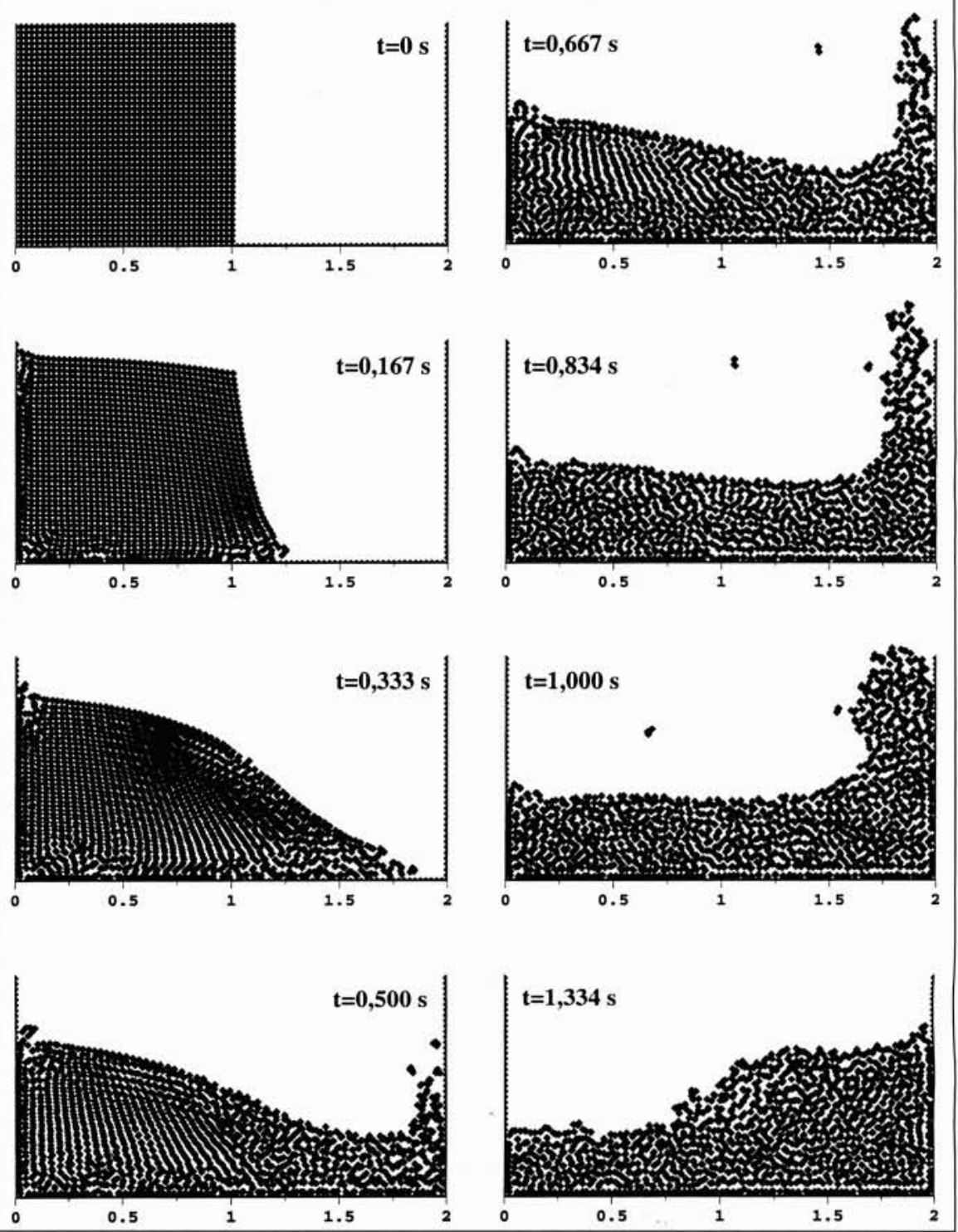

1. Impact d'une vague sur un mur (1600 particules). Evolution de la position des particules au cours du temps.

construction des outils de résolution, d'analyse, et d'aide à la décision ; souhaitons que l'école française de l'eau sache jouer sa carte et se montrer à la hauteur de ses ancêtres dont les noms émaillent les manuels de mécanique des fluides.

\section{Bibliographie}

[1] JAMES Gleick : La théorie du chaos. Flammarion 1989.

[2] JeAn-Michel. Hervouet : TElemaC, a hydroinformatic system. La Houille Blanche. $\mathrm{n}^{\circ} 3 / 4-1999$

[3] JEAN-Michel. HeRvouet, Lucas van Haren : Recent advances in numerical methods for fluid flow. In M.G. ANDERSON, D.E. Walling and P.D. Bates (eds.) Floodplain processes. John Wiley, Chichester. 1995

[4] Tom Demarco, Timothy Lister : Peopleware, productive projects and teams. Dorset House Publishing. 1987

[5] Frederick P. Brooks Jr. : The mythical man-month. Essays on software engineering. Addison Wesley 1982. 